\title{
Adding hypnosis to cognitive behavioural therapy may reduce some acute stress disorder symptoms
}

Bryant RA, Moulds ML, Guthrie RM, et al. The additive benefit of hypnosis and cognitive-behavioral therapy in treating acute stress disorder. J Consult Clin Psychol 2005;73:334-40.

\section{Does adding hypnotherapy to cognitive behavioural therapy (CBT) reduce the symptoms of acute stress disorder compared with CBT or supportive counselling alone?}

\section{METHODS}

Design: Randomised controlled trial.

Allocation: Not concealed.

Blinding: Assessors blinded, nature of intervention precluded participant blinding.

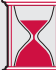

Follow up period: Six months

Setting: Hospital post-traumatic stress disorder (PTSD) unit Sydney, Australia; time period not stated.

Patients: Eighty seven civilians aged $17-60$ years, with acute stress disorder (ASD; DSM-IV criteria) subsequent to a motor vehicle accident (39 people) or non-sexual assault (48 people). Main exclusion criteria: history of childhood sexual abuse, psychosis, or substance abuse; organic brain disorder; or current suicidal thoughts.

$\mathbf{R}_{\mathbf{x}}$

Intervention: Cognitive behavioural therapy (CBT) alone: five weekly 90 minute sessions involving imaginal and in vivo exposure, cognitive restructuring, and relapse prevention techniques. Participants completed daily homework to reinforce the concepts learnt during the sessions. CBT plus hypnosis: as for CBT alone, but with the use of a hypnotic induction audiotape before imaginal exposure. Supportive counselling: provided unconditional support for participant and education about trauma and problem solving skills using the same schedule as CBT. Exposure, cognitive restructuring, and hypnosis techniques were avoided. Participants noted moods and problems experienced in a homework diary.

Outcomes: Impact of event scale (IES), Beck depression scale-2 (BDI-2), Clinical administered PTSD scale-2 (CAPS-2; used for diagnosis of PTSD), Beck Anxiety Inventory (BAI).

Patient follow up: $79 \%$ overall (73\% in CBT alone group, $77 \%$ in CBT-hypnosis group, $92 \%$ in SC group; $p=0.19$ for difference).

\section{MAIN RESULTS}

Cognitive behavioural therapy (CBT) plus hypnosis improved reexperiencing symptoms (IES-intrusion subscale) more than CBT alone $(\mathrm{p}<0.05)$ or supportive counselling $(\mathrm{SC}, \mathrm{p}<0.005)$ at five weeks (mean scores: 11.3 CBT plus hypnosis $v$ 16.6 CBT alone $v 19.8 \mathrm{SC}$ ). The difference between CBT-hypnosis and SC remained significant $(\mathrm{p}<0.05)$ at six months, but the difference between CBT-hypnosis and CBT alone did not (mean scores: 13.6 CBT plus hypnosis $v 17.0$ CBT alone $v 20.2$ SC). Treatment groups did not differ significantly on any other symptom measure in intention to treat analyses. There was

For correspondence: Richard A Bryant, School of Psychology, University of New South Wales, New South Wales 2052, Sydney, Australia;

r.bryant@unsw.edu.au

Source of funding: National Health and Medical Research Council, Australia no significant difference between treatments in the proportion of participants diagnosed with PTSD at up to six months (AR at five weeks: $36 \%$ CBT alone $v 30 \%$ with CBT-hypnosis $v 50 \%$ with SC; at six months: $42 \%$ with CBT alone $v 40 \%$ with CBT-hypnosis $v 58 \%$ with $\mathrm{SC}$; p value reported as not significant).

\section{CONCLUSIONS}

Adding hypnosis to CBT may improve the re-experiencing symptoms of acute stress disorder more than CBT alone or supportive counselling at five weeks, but long term effects and effects on other symptoms are less clear.

\section{NOTES}

Once participants were randomised using a random numbers system the study author "amended allocation to ensure that gender, trauma type, and PTSD severity were balanced across groups". This may have resulted in imbalance between groups for other potential confounders.

Commentary

A cute stress disorder (ASD) represents what most clinicians would recognise as an extreme emotional response within a month of a traumatic event. In many individuals it is a precursor to the development of chronic post traumatic stress disorder (PTSD). In previous work ${ }^{1}$ Bryant et al showed that five 90 minute sessions of trauma focused cognitive behavioural therapy (CBT) was significantly better at preventing the development of PTSD in ASD sufferers than supportive counselling. This, along with other research, has resulted in trauma focused CBT being widely advocated as the early intervention of choice for ASD sufferers and other individuals who experience marked traumatic stress symptoms within three months of a traumatic event. ${ }^{2}$

Unfortunately trauma focused CBT does not help all ASD sufferers, and it is important that attempts are made to identify more effective treatments. The current study is a well designed trial that suggests hypnosis may accelerate the positive effects of trauma focused CBT on reexperiencing symptoms. However, the apparent difference between trauma focused CBT alone and trauma focused CBT plus hypnosis disappeared at follow up and, given the relatively small sample size and absence of effects on other symptoms or PTSD diagnosis, one is left wondering how significant the finding really is. It is also of concern that around a quarter of those in both the control and intervention groups did not complete treatment.

The results of this trial are unlikely to change clinical practice or the treatment guidelines for ASD. However, the results confirm that the addition of hypnosis to trauma focused CBT warrants further study. I would support the authors' call for further research considering the addition of hypnosis to trauma focused CBT within a broader framework. Work on how to make trauma focused CBT more acceptable to ASD sufferers is also required.

Dr Jonathan I Bisson DM MRCPsych Clinical Senior Lecturer, Cardiff University, Cardiff, UK

1 Bryant RA, Moulds ML, Nixon RVD. Cognitive behaviour therapy of acute stress disorder: A four-year follow-up. Behav Res Ther 2003;41:489-94.

2 National Collaborating Centre for Mental Health. Post-traumatic stress disorder: The management of PTSD in adults and children in primary and secondary care. London and Leicester: Gaskell and BPS, 2005. 